\title{
Oscillation of a class of second-order linear impulsive differential equations
}

\section{Jessada Tariboon* and Phollakrit Thiramanus}

\section{"Correspondence:}

jessadat@kmutnb.ac.th

Department of Mathematics,

Faculty of Applied Science, King

Mongkut's University of Technology

North Bangkok, Bangkok, 10800,

Thailand

\section{Abstract}

In this paper, we investigate the oscillation of a class of second-order linear impulsive differential equations of the form

$$
\left\{\begin{array}{l}
\left(a(t)\left[x^{\prime}(t)+\lambda x(t)\right]\right)^{\prime}+p(t) x(t)=0, \quad t \geq t_{0}, t \neq t_{k} \\
x\left(t_{k}^{+}\right)=b_{k} x\left(t_{k}\right), \quad x^{\prime}\left(t_{k}^{+}\right)=c_{k} x^{\prime}\left(t_{k}\right), \quad k=1,2, \ldots
\end{array}\right.
$$

By using the equivalence transformation and the associated Riccati techniques, some interesting results are obtained.

MSC: $34 \mathrm{~A} 37 ; 34 \mathrm{C} 10$

Keywords: oscillation; nonoscillation; impulsive differential equation

\section{Introduction}

Impulsive differential equations are recognized as adequate mathematical models for studying evolution processes that are subject to abrupt changes in their states at certain moments. Many applications in physics, biology, control theory, economics, applied sciences and engineering exhibit impulse effects (see [1-4]). In recent years, the study of the oscillation of all solutions of impulsive differential equations have been the subject of many research works. See, for example, [5-11] and the references cited therein.

In this article, we consider the second-order linear impulsive differential equation of the form

$$
\left\{\begin{array}{l}
\left(a(t)\left[x^{\prime}(t)+\lambda x(t)\right]\right)^{\prime}+p(t) x(t)=0, \quad t \geq t_{0}, t \neq t_{k}, \\
x\left(t_{k}^{+}\right)=b_{k} x\left(t_{k}\right), \quad x^{\prime}\left(t_{k}^{+}\right)=c_{k} x^{\prime}\left(t_{k}\right), \quad k=1,2, \ldots,
\end{array}\right.
$$

where $0 \leq t_{0}<t_{1}<\cdots<t_{k} \rightarrow \infty, \lim _{k \rightarrow \infty} t_{k}=+\infty, a(t) \in C\left(\left[t_{0}, \infty\right),(0, \infty)\right)$ and $p(t) \in$ $C\left(\left[t_{0}, \infty\right), \mathbb{R}\right),\left\{b_{k}\right\}$ and $\left\{c_{k}\right\}$ are two known sequences of positive real numbers, $\lambda$ is a real number, and

$$
\begin{aligned}
& x^{\prime}\left(t_{k}\right)=x^{\prime}\left(t_{k}^{-}\right)=\lim _{h \rightarrow 0^{-}} \frac{x\left(t_{k}+h\right)-x\left(t_{k}\right)}{h}, \\
& x^{\prime}\left(t_{k}^{+}\right)=\lim _{h \rightarrow 0^{+}} \frac{x\left(t_{k}+h\right)-x\left(t_{k}^{+}\right)}{h} .
\end{aligned}
$$

(c) 2012 Tariboon and Thiramanus; licensee Springer. This is an Open Access article distributed under the terms of the Creative Commons Attribution License (http://creativecommons.org/licenses/by/2.0), which permits unrestricted use, distribution, and reproduction in any medium, provided the original work is properly cited. 
Let $J \subset \mathbb{R}$ be an interval and $P C(J, \mathbb{R})=\{x: J \rightarrow \mathbb{R}: x(t)$ be continuous everywhere except at some $t_{k}$ at which $x\left(t_{k}^{+}\right)$and $x\left(t_{k}^{-}\right)$exist and $\left.x\left(t_{k}^{-}\right)=x\left(t_{k}\right)\right\}$.

A function $x \in P C\left(\left[t_{0}, \infty\right), \mathbb{R}\right)$ is said to be a solution of Eq. (1.1) if

(i) $x(t)$ satisfies $\left(a(t)\left[x^{\prime}(t)+\lambda x(t)\right]\right)^{\prime}+p(t) x(t)=0$ for $t \in\left[t_{0}, \infty\right)$ and $t \neq t_{k}$,

(ii) $x\left(t_{k}^{+}\right)=b_{k} x\left(t_{k}\right), x^{\prime}\left(t_{k}^{+}\right)=c_{k} x^{\prime}\left(t_{k}\right)$ for each $t_{k}$, and $x(t)$ and $x^{\prime}(t)$ are left continuous for each $t_{k}, k=1,2, \ldots$.

Definition 1.1 A nontrivial solution of Eq. (1.1) is said to be nonoscillatory if the solution is eventually positive or eventually negative. Otherwise, it is said to be oscillatory. Eq. (1.1) is said to be oscillatory if all solutions are oscillatory.

If $\lambda=0$, then Eq. (1.1) reduces to the second-order linear differential equation with impulses

$$
\left\{\begin{array}{l}
\left(a(t) x^{\prime}(t)\right)^{\prime}+p(t) x(t)=0, \quad t \geq t_{0}, t \neq t_{k}, \\
x\left(t_{k}^{+}\right)=b_{k} x\left(t_{k}\right), \quad x^{\prime}\left(t_{k}^{+}\right)=c_{k} x^{\prime}\left(t_{k}\right), \quad k=1,2, \ldots
\end{array}\right.
$$

In [12] Luo et al. and [13] Guo et al. gave some excellent results on the oscillation and nonoscillation of Eq. (1.2) by using associated Riccati techniques and an equivalence transformation. Moreover, Luo et al. showed that the oscillation of Eq. (1.2) can be caused by impulsive perturbations, though the corresponding equation without impulses admits a nonoscillatory solution.

If $a(t) \equiv 1$ and $\lambda \neq 0$, then Eq. (1.1) reduces to the impulsive Langevin equation of the form

$$
\left\{\begin{array}{l}
\frac{d}{d t}\left(\frac{d}{d t}+\lambda\right) x(t)+p(t) x(t)=0, \quad t \geq t_{0}, t \neq t_{k} \\
x\left(t_{k}^{+}\right)=b_{k} x\left(t_{k}\right), \quad x^{\prime}\left(t_{k}^{+}\right)=c_{k} x^{\prime}\left(t_{k}\right), \quad k=1,2, \ldots
\end{array}\right.
$$

The Langevin equation (first formulated by Langevin in 1908) is found to be an effective tool to describe the evolution of physical phenomena in fluctuating environments. For more details of the Langevin equation without impulses and its applications, we refer the reader to [14].

If $\lambda=0$ and $b_{k}=c_{k}=1$ for all $k=1,2, \ldots$, then Eq. (1.1) reduces to the self-adjoint secondorder differential equation

$$
(a(t) x(t))^{\prime}+p(t) x(t)=0, \quad t>t_{0} .
$$

There are many good results on the oscillation and nonoscillation of Eq. (1.4); see, for example, [15-18].

\section{Main results}

Now we are in the position to establish the main result.

Lemma 2.1 If the second-order differential equation

$$
\left[\left(\prod_{T \leq t_{k}<t} d_{k}^{-1}\right) a(t)\left\{y^{\prime}(t)+\lambda\left(1-\frac{2}{a(t)} \sum_{T \leq t_{k}<t} \prod_{t_{k}<t_{j}<t} d_{j}\left(1-d_{k}\right) a\left(t_{k}\right)\right) y(t)\right\}\right]^{\prime}
$$




$$
\begin{aligned}
& +\left(\prod_{T \leq t_{k}<t} d_{k}^{-1}\right)\left[p(t)+\frac{\lambda^{2}}{a(t)}\left(\sum_{T \leq t_{k}<t} \prod_{t_{k}<t_{j}<t} d_{j}\left(1-d_{k}\right) a\left(t_{k}\right)\right)^{2}\right. \\
& \left.-\lambda^{2} \sum_{T \leq t_{k}<t} \prod_{t_{k}<t_{j}<t} d_{j}\left(1-d_{k}\right) a\left(t_{k}\right)\right] y(t)=0, \quad t>T,
\end{aligned}
$$

is oscillatory, then Eq. (1.1) is oscillatory, where $d_{k}=c_{k} / b_{k}, k=1,2, \ldots$.

Proof For the sake of contradiction, suppose that Eq. (1.1) has an eventually positive solution $x(t)$. Then there exists a constant $T \geq t_{0}$ such that $x(t)>0$ for $t \geq T$.

We let

$$
u(t)=\frac{a(t)\left(e^{\lambda t} x(t)\right)^{\prime}}{e^{\lambda t} x(t)}=\frac{a(t)\left(x^{\prime}(t)+\lambda x(t)\right)}{x(t)}, \quad t \geq T
$$

Then

$$
\begin{aligned}
u^{\prime}(t)= & \frac{e^{\lambda t} x(t)\left[a(t)\left(e^{\lambda t} x(t)\right)^{\prime}\right]^{\prime}-a(t)\left[\left(e^{\lambda t} x(t)\right)^{\prime}\right]^{2}}{\left(e^{\lambda t} x(t)\right)^{2}} \\
= & \frac{\left[a(t)\left(e^{\lambda t} x(t)\right)^{\prime}\right]^{\prime}}{e^{\lambda t} x(t)}-\frac{a(t)\left[\left(e^{\lambda t} x(t)\right)^{\prime}\right]^{2}}{\left(e^{\lambda t} x(t)\right)^{2}} \\
= & \frac{\left(e^{\lambda t}\left[a(t)\left(x^{\prime}(t)+\lambda x(t)\right)\right]\right)^{\prime}}{e^{\lambda t} x(t)}-\frac{a(t)\left[\left(e^{\lambda t} x(t)\right)^{\prime}\right]^{2}}{\left(e^{\lambda t} x(t)\right)^{2}} \\
= & \frac{\left(a(t)\left(x^{\prime}(t)+\lambda x(t)\right)\right)^{\prime}}{x(t)}+\lambda \frac{a(t)\left(x^{\prime}(t)+\lambda x(t)\right)}{x(t)} \\
& -\frac{a(t)\left[\left(e^{\lambda t} x(t)\right)^{\prime}\right]^{2}}{\left(e^{\lambda t} x(t)\right)^{2}} \\
= & -p(t)+\lambda u(t)-\frac{u^{2}(t)}{a(t)}
\end{aligned}
$$

can be obtained. Therefore,

$$
u^{\prime}(t)-\lambda u(t)+\frac{u^{2}(t)}{a(t)}+p(t)=0, \quad t \geq T, t \neq t_{k} .
$$

On the other hand, we have

$$
u\left(t_{k}^{+}\right)=\frac{a\left(t_{k}^{+}\right)\left(x^{\prime}\left(t_{k}^{+}\right)+\lambda x\left(t_{k}^{+}\right)\right)}{x\left(t_{k}^{+}\right)}=\frac{a\left(t_{k}\right)\left(c_{k} x^{\prime}\left(t_{k}\right)+\lambda b_{k} x\left(t_{k}\right)\right)}{b_{k} x\left(t_{k}\right)} .
$$

Let $e_{k}=\lambda\left(1-d_{k}\right) a\left(t_{k}\right)$, then we get

$$
u\left(t_{k}^{+}\right)=d_{k} u\left(t_{k}\right)+e_{k}, \quad t_{k} \geq T, k=1,2, \ldots
$$

Now, we define

$$
v(t)=\prod_{T \leq t_{k}<t} d_{k}^{-1}\left[u(t)-\sum_{T \leq t_{k}<t} \prod_{t_{k}<t_{j}<t} d_{j} e_{k}\right], \quad t>T .
$$


Then, for $t_{n}>T$, we get that

$$
\begin{aligned}
v\left(t_{n}^{+}\right) & =\prod_{T \leq t_{k} \leq t_{n}} d_{k}^{-1}\left[u\left(t_{n}^{+}\right)-\sum_{T \leq t_{k} \leq t_{n}} \prod_{t_{k}<t_{j} \leq t_{n}} d_{j} e_{k}\right] \\
& =\prod_{T \leq t_{k} \leq t_{n}} d_{k}^{-1}\left[d_{n} u\left(t_{n}\right)+e_{n}-\sum_{T \leq t_{k}<t_{n}} \prod_{t_{k}<t_{j}<t_{n}} d_{j} d_{n} e_{k}-e_{n}\right] \\
& =\prod_{T \leq t_{k}<t_{n}} d_{k}^{-1}\left[u\left(t_{n}\right)-\sum_{T \leq t_{k}<t_{n}} \prod_{t_{k}<t_{j}<t_{n}} d_{j} e_{k}\right] \\
& =v\left(t_{n}\right) .
\end{aligned}
$$

Therefore, $v(t)$ is continuous on $(T, \infty)$.

We have

$$
\left(\prod_{T \leq t_{k}<t} d_{k}^{-1}\right) \times\left(\sum_{T \leq t_{k}<t} \prod_{t_{k}<t_{j}<t} d_{j} e_{k}\right)=\sum_{T \leq t_{k}<t} \prod_{T \leq t_{j} \leq t_{k}} d_{j}^{-1} e_{k} .
$$

Then, for $t>T, t \neq t_{n}$ and from (2.2), we get that

$$
\begin{aligned}
& v^{\prime}(t)=\lim _{h \rightarrow 0}\left(\prod_{T \leq t_{k}<t+h} d_{k}^{-1}\left[u(t+h)-\sum_{T \leq t_{k}<t+h} \prod_{t_{k}<t_{j}<t+h} d_{j} e_{k}\right]\right. \\
& \left.-\prod_{T \leq t_{k}<t} d_{k}^{-1}\left[u(t)-\sum_{T \leq t_{k}<t} \prod_{t_{k}<t_{j}<t} d_{j} e_{k}\right]\right) / h \\
& =\lim _{h \rightarrow 0} \frac{\prod_{T \leq t_{k}<t+h} d_{k}^{-1} u(t+h)-\prod_{T \leq t_{k}<t} d_{k}^{-1} u(t)}{h} \\
& =\left(\prod_{T \leq t_{k}<t} d_{k}^{-1}\right) \lim _{h \rightarrow 0} \frac{u(t+h)-u(t)}{h} \\
& =\left(\prod_{T \leq t_{k}<t} d_{k}^{-1}\right) u^{\prime}(t)=\left(\prod_{T \leq t_{k}<t} d_{k}^{-1}\right)\left[\lambda u(t)-\frac{u^{2}(t)}{a(t)}-p(t)\right] \\
& =\left(\prod_{T \leq t_{k}<t} d_{k}^{-1}\right)\left\{\lambda\left[v(t)\left(\prod_{T \leq t_{k}<t} d_{k}\right)+\sum_{T \leq t_{k}<t} \prod_{t_{k}<t_{j}<t} d_{j} e_{k}\right]\right. \\
& \left.-\frac{1}{a(t)}\left[v(t)\left(\prod_{T \leq t_{k}<t} d_{k}\right)+\sum_{T \leq t_{k}<t} \prod_{t_{k}<t_{j}<t} d_{j} e_{k}\right]^{2}-p(t)\right\} \\
& =\lambda v(t)+\lambda \sum_{T \leq t_{k}<t} \prod_{T \leq t_{j} \leq t_{k}} d_{j}^{-1} e_{k}-\left(\prod_{T \leq t_{k}<t} d_{k}\right) \frac{v^{2}(t)}{a(t)} \\
& -2 \frac{v(t)}{a(t)} \sum_{T \leq t_{k}<t} \prod_{t_{k}<t_{j}<t} d_{j} e_{k}-\frac{1}{a(t)}\left(\prod_{T \leq t_{k}<t} d_{k}^{-1}\right) \\
& \times\left(\sum_{T \leq t_{k}<t} \prod_{t_{k}<t_{j}<t} d_{j} e_{k}\right)^{2}-\left(\prod_{T \leq t_{k}<t} d_{k}^{-1}\right) p(t) .
\end{aligned}
$$


The left-hand and the right-hand derivatives of $v(t)$ at $t=t_{n}$ are given by

$$
\begin{aligned}
& v^{\prime}\left(t_{n}^{-}\right)=\left(\prod_{T \leq t_{k}<t_{n}} d_{k}^{-1}\right) u^{\prime}\left(t_{n}^{-}\right)=\left(\prod_{T \leq t_{k}<t_{n}} d_{k}^{-1}\right) u^{\prime}\left(t_{n}\right) \\
& v^{\prime}\left(t_{n}^{+}\right)=\left(\prod_{T \leq t_{k} \leq t_{n}} d_{k}^{-1}\right) u^{\prime}\left(t_{n}^{+}\right)=\left(\prod_{T \leq t_{k} \leq t_{n}} d_{k}^{-1}\right) d_{n} u^{\prime}\left(t_{n}\right)=\left(\prod_{T \leq t_{k}<t_{n}} d_{k}^{-1}\right) u^{\prime}\left(t_{n}\right) .
\end{aligned}
$$

Hence, for $t=t_{n}$, we have

$$
\begin{aligned}
& v^{\prime}\left(t_{n}\right)=\left(\prod_{T \leq t_{k}<t_{n}} d_{k}^{-1}\right) u^{\prime}\left(t_{n}\right)=\left(\prod_{T \leq t_{k}<t_{n}} d_{k}^{-1}\right) \lim _{t \rightarrow t_{n}^{-}} u^{\prime}(t) \\
& =\left(\prod_{T \leq t_{k}<t_{n}} d_{k}^{-1}\right) \lim _{t \rightarrow t_{n}^{-}}\left[\lambda u(t)-\frac{u^{2}(t)}{a(t)}-p(t)\right] \\
& =\left(\prod_{T \leq t_{k}<t_{n}} d_{k}^{-1}\right)\left[\lambda u\left(t_{n}\right)-\frac{u^{2}\left(t_{n}\right)}{a\left(t_{n}\right)}-p\left(t_{n}\right)\right] \\
& =\left(\prod_{T \leq t_{k}<t_{n}} d_{k}^{-1}\right)\left\{\lambda\left[v\left(t_{n}\right)\left(\prod_{T \leq t_{k}<t_{n}} d_{k}\right)+\sum_{T \leq t_{k}<t_{n}} \prod_{t_{k}<t_{j}<t_{n}} d_{j} e_{k}\right]\right. \\
& \left.-\frac{1}{a\left(t_{n}\right)}\left[v\left(t_{n}\right)\left(\prod_{T \leq t_{k}<t_{n}} d_{k}\right)+\sum_{T \leq t_{k}<t_{n}} \prod_{t_{k}<t_{j}<t_{n}} d_{j} e_{k}\right]^{2}-p\left(t_{n}\right)\right\} \\
& =\lambda v\left(t_{n}\right)+\lambda \sum_{T \leq t_{k}<t_{n}} \prod_{T \leq t_{j} \leq t_{k}} d_{j}^{-1} e_{k}-\left(\prod_{T \leq t_{k}<t_{n}} d_{k}\right) \frac{v^{2}\left(t_{n}\right)}{a\left(t_{n}\right)} \\
& -2 \frac{v\left(t_{n}\right)}{a\left(t_{n}\right)} \sum_{T \leq t_{k}<t_{n}} \prod_{t_{k}<t_{j}<t_{n}} d_{j} e_{k}-\frac{1}{a\left(t_{n}\right)}\left(\prod_{T \leq t_{k}<t_{n}} d_{k}^{-1}\right) \\
& \times\left(\sum_{T \leq t_{k}<t_{n}} \prod_{t_{k}<t_{j}<t_{n}} d_{j} e_{k}\right)^{2}-\left(\prod_{T \leq t_{k}<t_{n}} d_{k}^{-1}\right) p\left(t_{n}\right) .
\end{aligned}
$$

Thus,

$$
\begin{aligned}
& v^{\prime}(t)+\left(\prod_{T \leq t_{k}<t} d_{k}\right) \frac{v^{2}(t)}{a(t)}-\lambda v(t)+2 \frac{v(t)}{a(t)} \sum_{T \leq t_{k}<t} \prod_{t_{k}<t_{j}<t} d_{j} e_{k} \\
& -\lambda \sum_{T \leq t_{k}<t} \prod_{T \leq t_{j} \leq t_{k}} d_{j}^{-1} e_{k}+\frac{1}{a(t)}\left(\prod_{T \leq t_{k}<t} d_{k}^{-1}\right)\left(\sum_{T \leq t_{k}<t} \prod_{t_{k}<t_{j}<t} d_{j} e_{k}\right)^{2} \\
& +\left(\prod_{T \leq t_{k}<t} d_{k}^{-1}\right) p(t)=0, \quad t>T .
\end{aligned}
$$

We set

$$
w(t)=\exp \left\{\int_{T}^{t}\left[\left(\prod_{T \leq t_{k}<s} d_{k}\right) \frac{v(s)}{a(s)}-\lambda+\frac{2}{a(s)} \sum_{T \leq t_{k}<s} \prod_{t_{k}<t_{j}<s} d_{j} e_{k}\right] d s\right\}, \quad t>T .
$$


Then, $w(t)>0$ for $t>T$ and

$$
w^{\prime}(t)=w(t)\left[\left(\prod_{T \leq t_{k}<t} d_{k}\right) \frac{v(t)}{a(t)}-\lambda+\frac{2}{a(t)} \sum_{T \leq t_{k}<t} \prod_{t_{k}<t_{j}<t} d_{j} e_{k}\right] .
$$

From (2.5), we obtain

$$
\begin{aligned}
& {\left[\left(\prod_{T \leq t_{k}<t} d_{k}^{-1}\right) a(t) w^{\prime}(t)+\lambda\left(\prod_{T \leq t_{k}<t} d_{k}^{-1}\right) a(t) w(t)-2 \sum_{T \leq t_{k}<t} \prod_{T \leq t_{j} \leq t_{k}} d_{j}^{-1} e_{k} w(t)\right]^{\prime}} \\
& =w(t) v^{\prime}(t)+v(t) w^{\prime}(t) \\
& =w(t)\left[v^{\prime}(t)+\left(\prod_{T \leq t_{k}<t} d_{k}\right) \frac{v^{2}(t)}{a(t)}-\lambda v(t)+2 \frac{v(t)}{a(t)} \sum_{T \leq t_{k}<t} \prod_{t_{k}<t_{j}<t} d_{j} e_{k}\right] \\
& =w(t)\left[\lambda \sum_{T \leq t_{k}<t} \prod_{T \leq t_{j} \leq t_{k}} d_{j}^{-1} e_{k}-\frac{1}{a(t)}\left(\prod_{T \leq t_{k}<t} d_{k}^{-1}\right)\right. \\
& \left.\quad \times\left(\sum_{T \leq t_{k}<t} \prod_{t_{k}<t_{j}<t} d_{j} e_{k}\right)^{2}-\left(\prod_{T \leq t_{k}<t} d_{k}^{-1}\right) p(t)\right] .
\end{aligned}
$$

Therefore,

$$
\begin{aligned}
& {\left[\left(\prod_{T \leq t_{k}<t} d_{k}^{-1}\right) a(t)\left\{w^{\prime}(t)+\lambda\left(1-\frac{2}{a(t)} \sum_{T \leq t_{k}<t} \prod_{t_{k}<t_{j}<t} d_{j}\left(1-d_{k}\right) a\left(t_{k}\right)\right) w(t)\right\}\right]^{\prime}} \\
& \quad+\left(\prod_{T \leq t_{k}<t} d_{k}^{-1}\right)\left[p(t)+\frac{\lambda^{2}}{a(t)}\left(\sum_{T \leq t_{k}<t} \prod_{t_{k}<t_{j}<t} d_{j}\left(1-d_{k}\right) a\left(t_{k}\right)\right)^{2}\right. \\
& \left.\quad-\lambda^{2} \sum_{T \leq t_{k}<t} \prod_{t_{k}<t_{j}<t} d_{j}\left(1-d_{k}\right) a\left(t_{k}\right)\right] w(t)=0, \quad t>T .
\end{aligned}
$$

This implies that $w(t)$ is an eventually positive solution of Eq. (2.1) which is a contradiction. A similar argument can be used to prove that Eq. (2.1) cannot have an eventually negative solution. Therefore, from Definition 1.1, the solution of Eq. (2.1) is oscillatory. The proof is complete.

Lemma 2.2 (Leighton type oscillation criteria) Assume that the functions $g(t), q(t) \in$ $P C\left(\left[t_{0}, \infty\right), \mathbb{R}\right)$ and $h(t) \in P C\left(\left[t_{0}, \infty\right),(0, \infty)\right)$.

If

$$
\int_{T}^{\infty} g(s) e^{-\int_{T}^{s} q(\sigma) d \sigma} d s=\infty \quad \text { and } \quad \int_{T}^{\infty} \frac{1}{h(s)} e^{\int_{T}^{s} q(\sigma) d \sigma} d s=\infty
$$

then

$$
\left(h(t)\left[y^{\prime}(t)+q(t) y(t)\right]\right)^{\prime}+g(t) y(t)=0
$$

is oscillatory. 
Proof Let $y(t)$ be a nonoscillatory solution of the Eq. (2.6). Without loss of generality, we assume that there exists a $T \geq t_{0}$ such that $y(t)>0$ for $t \geq T$. We define

$$
u(t)=\frac{h(t)\left(e^{\int_{T}^{t} q(\sigma) d \sigma} y(t)\right)^{\prime}}{e^{\int_{T}^{t} q(\sigma) d \sigma} y(t)}, \quad t \geq T
$$

Then the equation

$$
u^{\prime}(t)-q(t) u(t)+\frac{u^{2}(t)}{h(t)}+g(t)=0
$$

has a solution $u(t)$ on $[T, \infty)$. It is easy to see that the solution of Eq. (2.7) satisfies the following equation:

$$
\begin{aligned}
u(t)= & e^{\int_{T}^{t} q(\sigma) d \sigma} u(T)-e^{\int_{T}^{t} q(\sigma) d \sigma} \int_{T}^{t} \frac{u^{2}(s)}{h(s)} e^{-\int_{T}^{s} q(\sigma) d \sigma} d s \\
& -e^{\int_{T}^{t} q(\sigma) d \sigma} \int_{T}^{t} g(s) e^{-\int_{T}^{s} q(\sigma) d \sigma} d s .
\end{aligned}
$$

Since $\int_{T}^{\infty} g(s) e^{-\int_{T}^{s} q(\sigma) d \sigma} d s=\infty$, then there exists $\tau>T$ such that

$$
u(T)-\int_{T}^{t} g(s) e^{-\int_{T}^{s} q(\sigma) d \sigma} d s<0
$$

for all $t$ in $[\tau, \infty)$. Hence, from (2.8), it follows that

$$
u(t)<-e^{\int_{T}^{t} q(s) d s} \int_{T}^{t} \frac{u^{2}(s)}{h(s)} e^{-\int_{T}^{s} q(\sigma) d \sigma} d s, \quad t \in[\tau, \infty) .
$$

Let

$$
r(t)=\int_{T}^{t} \frac{u^{2}(s)}{h(s)} e^{-\int_{T}^{s} q(\sigma) d \sigma} d s, \quad t \in[\tau, \infty)
$$

Then $u(t)<-r(t) e^{\int_{T}^{t} q(\sigma) d \sigma}$ and

$$
r^{\prime}(t)=\frac{u^{2}(t)}{h(t)} e^{-\int_{T}^{t} q(\sigma) d \sigma}>\frac{r^{2}(t)}{h(t)} e^{e_{T}^{t} q(\sigma) d \sigma}, \quad t \in[\tau, \infty)
$$

Integrating (2.9) from $\tau>T$ to $\infty$, we obtain

$$
-\frac{1}{r(\infty)}+\frac{1}{r(\tau)}>\int_{\tau}^{\infty} \frac{1}{h(s)} e^{\int_{T}^{s} q(\sigma) d \sigma} d s .
$$

Hence,

$$
\int_{\tau}^{\infty} \frac{1}{h(s)} e^{\int_{T}^{s} q(\sigma) d \sigma} d s<\frac{1}{r(\tau)}<\infty,
$$

which is a contradiction. Thus, the solution $y(t)$ is oscillatory. The proof is complete. 
Theorem 2.3 Assume that

$$
\begin{aligned}
& \int_{T}^{\infty}\left(\prod_{T \leq t_{k}<t} d_{k}^{-1}\right)\left[p(t)+\frac{\lambda^{2}}{a(t)}\left(\sum_{T \leq t_{k}<t} \prod_{t_{k}<t_{j}<t} d_{j}\left(1-d_{k}\right) a\left(t_{k}\right)\right)^{2}\right. \\
& \left.-\lambda^{2} \sum_{T \leq t_{k}<t} \prod_{t_{k}<t_{j}<t} d_{j}\left(1-d_{k}\right) a\left(t_{k}\right)\right] \exp \left\{-\lambda \int_{T}^{t}\left(1-\frac{2}{a(s)}\right.\right. \\
& \left.\left.\quad \times \sum_{T \leq t_{k}<s} \prod_{t_{k}<t_{j}<s} d_{j}\left(1-d_{k}\right) a\left(t_{k}\right)\right) d s\right\} d t=\infty
\end{aligned}
$$

and

$$
\begin{aligned}
& \int_{T}^{\infty}\left(\prod_{T \leq t_{k}<t} d_{k}\right) \frac{1}{a(t)} \\
& \quad \times \exp \left\{\lambda \int_{T}^{t}\left(1-\frac{2}{a(s)} \times \sum_{T \leq t_{k}<s} \prod_{t_{k}<t_{j}<s} d_{j}\left(1-d_{k}\right) a\left(t_{k}\right)\right) d s\right\} d t=\infty,
\end{aligned}
$$

where $d_{k}=c_{k} / b_{k}, k=1,2, \ldots$ Then Eq. (1.1) is oscillatory.

If $b_{k}=c_{k}, k=1,2, \ldots$, then $d_{k}=1, k=1,2, \ldots$ and (1.1) becomes

$$
\left\{\begin{array}{l}
\left(a(t)\left[x^{\prime}(t)+\lambda x(t)\right]\right)^{\prime}+p(t) x(t)=0, \quad t \geq t_{0}, t \neq t_{k} \\
x\left(t_{k}^{+}\right)=b_{k} x\left(t_{k}\right), \quad x^{\prime}\left(t_{k}^{+}\right)=b_{k} x^{\prime}\left(t_{k}\right), \quad k=1,2, \ldots
\end{array}\right.
$$

Theorem 2.4 Eq. (2.12) is oscillatory if and only if

$$
\left(a(t)\left[y^{\prime}(t)+\lambda y(t)\right]\right)^{\prime}+p(t) y(t)=0, \quad t \geq t_{0},
$$

is oscillatory.

Proof From Lemma 2.1, we only need to prove that if Eq. (2.12) is oscillatory, then Eq. (2.13) is oscillatory.

Without loss of generality, we suppose that $y(t)$ is an eventually positive solution of (2.13) such that $y(t)>0$ for $t \geq T \geq t_{0}$. Set

$$
x(t)=\left(\prod_{T \leq t_{k}<t} b_{k}\right) y(t), \quad t>T .
$$

Then, for $t>T$, we have $x(t)>0$, and for $t_{n}>T$,

$$
x\left(t_{n}^{+}\right)=\left(\prod_{T \leq t_{k} \leq t_{n}} b_{k}\right) y\left(t_{n}^{+}\right)=b_{n}\left(\prod_{T \leq t_{k}<t_{n}} b_{k}\right) y\left(t_{n}\right)=b_{n} x\left(t_{n}\right) .
$$

Moreover, for $t \neq t_{n}>T$, we have

$$
x^{\prime}(t)=\left(\prod_{T \leq t_{k}<t} b_{k}\right) y^{\prime}(t)
$$


and

$$
x^{\prime}\left(t_{n}^{+}\right)=\left(\prod_{T \leq t_{k} \leq t_{n}} b_{k}\right) y^{\prime}\left(t_{n}^{+}\right)=b_{n}\left(\prod_{T \leq t_{k}<t_{n}} b_{k}\right) y^{\prime}\left(t_{n}\right)=b_{n} x^{\prime}\left(t_{n}\right)=c_{n} x^{\prime}\left(t_{n}\right) .
$$

Now we have for $t \neq t_{n}$

$$
\begin{aligned}
\left(a(t)\left[x^{\prime}(t)+\lambda x(t)\right]\right)^{\prime} & =\left(a(t)\left[\left(\prod_{T \leq t_{k}<t} b_{k}\right) y^{\prime}(t)+\lambda\left(\prod_{T \leq t_{k}<t} b_{k}\right) y(t)\right]\right)^{\prime} \\
& =\left(\prod_{T \leq t_{k}<t} b_{k}\right)\left(a(t)\left[y^{\prime}(t)+\lambda y(t)\right]\right)^{\prime} \\
& =-\left(\prod_{T \leq t_{k}<t} b_{k}\right) p(t) y(t)=-p(t) x(t) .
\end{aligned}
$$

Therefore,

$$
\left(a(t)\left[x^{\prime}(t)+\lambda x(t)\right]\right)^{\prime}+p(t) x(t)=0, \quad t \neq t_{n}, t>T .
$$

We get that $x(t)$ is an eventually positive solution of (2.12), a contradiction, and so the proof is complete.

Corollary 2.5 Assume that

$$
\int_{T}^{\infty} p(t) e^{-\lambda t} d t=\infty
$$

and

$$
\int_{T}^{\infty} \frac{e^{\lambda t}}{a(t)} d t=\infty
$$

Then Eq. (2.12) is oscillatory.

\section{Some examples}

In this section, we illustrate our results with two examples.

Example 3.1 Consider the following impulsive Langevin equation:

$$
\left\{\begin{array}{l}
\frac{d}{d t}\left(\frac{d}{d t}+\frac{2}{3}\right) x(t)+5^{t^{3}} x(t)=0, \quad t>0, t \neq k, \\
x\left(k^{+}\right)=\frac{k}{k+1} x(k), \quad x^{\prime}\left(k^{+}\right)=x^{\prime}(k), \quad k=1,2, \ldots
\end{array}\right.
$$

Set $d_{k}=\frac{k+1}{k}, \lambda=\frac{2}{3}, a(t)=a\left(t_{k}\right) \equiv 1$ and $p(t)=5^{t^{3}}$. If $T \in(m, m+1]$ for some integer $m \geq 0$, then we get

$$
\left(\prod_{T \leq t_{k}<[t]+1} \frac{k}{k+1}\right)=\frac{m+1}{m+2} \cdot \frac{m+2}{m+3} \cdots \frac{[t]}{[t]+1}=\frac{m+1}{[t]+1}
$$


and

$$
\begin{aligned}
\sum_{T \leq t_{k}<[t]} \prod_{t_{k}<t_{j}<[t]}\left(\frac{j+1}{j}\right)\left(\frac{1}{k}\right) \\
=\frac{1}{m+1} \cdot \frac{m+3}{m+2} \cdot \frac{m+4}{m+3} \cdots \frac{[t]}{[t]-1} \\
\quad+\frac{1}{m+2} \cdot \frac{m+4}{m+3} \cdot \frac{m+5}{m+4} \cdots \frac{[t]}{[t]-1}+\cdots+\frac{1}{[t]-2} \cdot \frac{[t]}{[t]-1}+\frac{1}{[t]-1} \\
=[t]\left(\frac{1}{m+1} \cdot \frac{1}{m+2}+\frac{1}{m+2} \cdot \frac{1}{m+3}+\cdots+\frac{1}{[t]-2} \cdot \frac{1}{[t]-1}\right)+\frac{1}{[t]-1} \\
=[t]\left(\frac{1}{m+1}-\frac{1}{[t]-1}\right)+\frac{1}{[t]-1} \\
=\frac{[t]}{m+1}-1,
\end{aligned}
$$

where $[\cdot]$ denotes the greatest integer function.

Hence,

$$
\begin{aligned}
& \int_{T}^{\infty}\left(\prod_{T \leq t_{k}<t} d_{k}^{-1}\right)\left[p(t)+\frac{\lambda^{2}}{a(t)}\left(\sum_{T \leq t_{k}<t} \prod_{t_{k}<t_{j}<t} d_{j}\left(1-d_{k}\right) a\left(t_{k}\right)\right)^{2}\right. \\
& \left.-\lambda^{2} \sum_{T \leq t_{k}<t} \prod_{t_{k}<t_{j}<t} d_{j}\left(1-d_{k}\right) a\left(t_{k}\right)\right] \exp \left\{-\lambda \int_{T}^{t}\left(1-\frac{2}{a(s)}\right.\right. \\
& \left.\left.\times \sum_{T \leq t_{k}<s} \prod_{t_{k}<t_{j}<s} d_{j}\left(1-d_{k}\right) a\left(t_{k}\right)\right) d s\right\} d t \\
& =\int_{T}^{\infty}\left(\prod_{T \leq t_{k}<t} \frac{k}{k+1}\right)\left[5^{t^{3}}+\left(\frac{2}{3}\right)^{2}\left(\sum_{T \leq t_{k}<t} \prod_{t_{k}<t_{j}<t}\left(\frac{j+1}{j}\right)\left(-\frac{1}{k}\right)\right)^{2}\right. \\
& \left.-\left(\frac{2}{3}\right)^{2} \sum_{T \leq t_{k}<t} \prod_{t_{k}<t_{j}<t}\left(\frac{j+1}{j}\right)\left(-\frac{1}{k}\right)\right] \exp \left\{-\frac{2}{3} \int_{T}^{t}(1-2\right. \\
& \left.\left.\times \sum_{T \leq t_{k}<s} \prod_{t_{k}<t_{j}<s}\left(\frac{j+1}{j}\right)\left(-\frac{1}{k}\right)\right) d s\right\} d t \\
& \geq \int_{T}^{\infty}\left(\prod_{T \leq t_{k}<[t]+1} \frac{k}{k+1}\right)\left[5^{t^{3}}+\left(\frac{2}{3}\right)^{2}\left(\sum_{T \leq t_{k}<[t]} \prod_{t_{k}<t_{j}<[t]}\left(\frac{j+1}{j}\right)\left(\frac{1}{k}\right)\right)\right. \\
& \left.\times\left(\sum_{T \leq t_{k}<[t]} \prod_{t_{k}<t_{j}<[t]}\left(\frac{j+1}{j}\right)\left(\frac{1}{k}\right)+1\right)\right] \exp \left\{-\frac{2}{3} \int_{T}^{t}(1+2\right. \\
& \left.\left.\times \sum_{T \leq t_{k}<[s]+1} \prod_{t_{k}<t_{j}<[s]+1}\left(\frac{j+1}{j}\right)\left(\frac{1}{k}\right)\right) d s\right\} d t \\
& \geq \int_{T}^{\infty}\left(\frac{m+1}{[t]+1}\right)\left[5^{t^{3}}+\left(\frac{2}{3}\right)^{2}\left(\frac{[t]}{m+1}-1\right)\right. \\
& \left.\times\left(\frac{[t]}{m+1}\right)\right] \exp \left\{-\frac{2}{3} \int_{T}^{t}\left(1+2\left(\frac{[s]+1}{m+1}-1\right)\right) d s\right\} d t
\end{aligned}
$$




$$
\begin{aligned}
& \geq \int_{T}^{\infty}\left(\frac{m+1}{t+1}\right)\left(5^{t^{3}}\right) \exp \left\{-\frac{2}{3} \int_{T}^{t}\left(1+2\left(\frac{s+1}{T}\right)\right) d s\right\} d t \\
& =\infty
\end{aligned}
$$

and

$$
\begin{aligned}
\int_{T}^{\infty} & \left(\prod_{T \leq t_{k}<t} d_{k}\right) \frac{1}{a(t)} \exp \left\{\lambda \int_{T}^{t}\left(1-\frac{2}{a(s)} \sum_{T \leq t_{k}<s} \prod_{t_{k}<t_{j}<s} d_{j}\left(1-d_{k}\right) a\left(t_{k}\right)\right) d s\right\} d t \\
= & \int_{T}^{\infty}\left(\prod_{T \leq t_{k}<t} \frac{k+1}{k}\right) \\
& \times \exp \left\{\frac{2}{3} \int_{T}^{t}\left(1+2 \sum_{T \leq t_{k}<s} \prod_{t_{k}<t_{j}<s} \frac{j+1}{j} \cdot \frac{1}{k}\right) d s\right\} d t \\
\geq & \int_{T}^{\infty}\left(\prod_{T \leq t_{k}<[t]} \frac{k+1}{k}\right) \exp \left\{\frac{2}{3} \int_{T}^{t}(1) d s\right\} d t \\
= & \int_{T}^{\infty}\left(\frac{m+2}{m+1} \cdot \frac{m+3}{m+2} \cdots \frac{[t]}{[t]-1}\right) \exp \left\{\frac{2}{3} \int_{T}^{t}(1) d s\right\} d t \\
= & \int_{T}^{\infty}\left(\frac{[t]}{m+1}\right) \exp \left\{\frac{2}{3}(t-T)\right\} d t \\
\geq & \int_{T}^{\infty}\left(\frac{t-1}{m+1}\right) \exp \left\{\frac{2}{3}(t-T)\right\} d t \\
= & \infty .
\end{aligned}
$$

By Theorem 2.3, Eq. (3.1) is oscillatory.

Example 3.2 Consider the equation

$$
\left\{\begin{array}{l}
\left(\frac{1}{\left(t^{2}+1\right)^{\frac{1}{2}}}\left[x^{\prime}(t)+\frac{3}{4} x(t)\right]\right)^{\prime}+\pi^{t} x(t)=0, \quad t \geq 0, t \neq t_{k}, \\
x\left(t_{k}^{+}\right)=b_{k} x\left(t_{k}\right), \quad x^{\prime}\left(t_{k}^{+}\right)=b_{k} x^{\prime}\left(t_{k}\right), \quad k=1,2, \ldots,
\end{array}\right.
$$

where $\left\{b_{k}\right\}$ is a known sequence of positive real numbers. It is easy to see that

$$
\int_{0}^{\infty} \pi^{t} e^{-\frac{3}{4} t} d t=\infty
$$

and

$$
\int_{0}^{\infty}\left(t^{2}+1\right)^{\frac{1}{2}} e^{\frac{3}{4} t} d t=\infty
$$

By Corollary 2.5, Eq. (3.2) is oscillatory.

The authors declare that they have no competing interests. 


\section{Acknowledgements}

This research work is financially supported by the Office of the Higher Education Commission of Thailand, and King Mongkut's University of Technology North Bangkok, Thailand.

Received: 15 August 2012 Accepted: 13 November 2012 Published: 27 November 2012

\section{References}

1. Lakshmikantham, V, Bainov, DD, Simeonov, PS: Theory of Impulsive Differential Equations. World Scientific, Singapore (1989)

2. Bainov, DD, Simeonov, PS: Impulsive Differential Equations: Periodic Solutions and Applications. Longman, Harlow (1993)

3. Bainov, DD, Simeonov, PS: Impulsive Differential Equations: Asymptotic Properties of the Solutions. World Scientific Singapore (1995)

4. Samoilenko, AM, Perestyuk, NA: Impulsive Differential Equations. World Scientific, Singapore (1995)

5. Agarwal, RP, Karakoç, F: A survey on oscillation of impulsive delay differential equations. Comput. Math. Appl. 60, 1648-1685 (2010)

6. Agarwal, RP, Karakoç, F, Zafer, A: A survey on oscillation of impulsive ordinary differential equations. Adv. Differ. Equ. 2010, Article ID 354841 (2010)

7. Guo, Z, Zhou, X, Ge, W: Interval oscillation criteria for second-order forced impulsive differential equations with mixed nonlinearities. J. Math. Anal. Appl. 381, 187-201 (2011)

8. Liu, Z, Sun, Y: Interval criteria for oscillation of a forced impulsive differential equation with Riemann-Stieltjes integral. Comput. Math. Appl. 63, 1577-1586 (2012)

9. Özbekler, A, Zafer, A: Leighton-Coles-Wintner type oscillation criteria for half-linear impulsive differential equations. Adv. Dyn. Syst. Appl. 5, 205-214 (2010)

10. Özbekler, A, Zafer, A: Nonoscillation and oscillation of second-order impulsive differential equations with periodic coefficients. Appl. Math. Lett. 25, 294-300 (2012)

11. Özbekler, A, Zafer, A: Oscillation of solutions of second order mixed nonlinear differential equations under impulsive perturbations. Comput. Math. Appl. 61, 933-940 (2011)

12. Luo, Z, Shen, J: Oscillation of second order linear differential equations with impulses. Appl. Math. Lett. 20, 75-81 (2007)

13. Guo, C, Xu, Z: On the oscillation of second order linear impulsive differential equations. Diff. Equ. Appl. 2, 319-330 (2010)

14. Coffey, WT, Kalmykov, YP, Waldron, JT: The Langevin Equation: With Applications to Stochastic Problems in Physics, Chemistry and Electrical Engineering. World Scientific, Singapore (2004)

15. Leighton, W: On self-adjoint differential equations of second order. J. Lon. Math. Soc. 27, $37-43$ (1952)

16. Swanson, GA: Comparison and Oscillation Theory of Linear Differential Equations. Academic Press, New York (1968)

17. Agarwal, RP, O'Regan, D: An Introduction to Ordinary Differential Equations. Springer, New York (2008)

18. Kelley, WG, Peterson, AC: The Theory of Differential Equations: Classical and Qualitative. Springer, New York (2010)

doi:10.1186/1687-1847-2012-205

Cite this article as: Tariboon and Thiramanus: Oscillation of a class of second-order linear impulsive differential equations. Advances in Difference Equations 2012 2012:205

\section{Submit your manuscript to a SpringerOpen ${ }^{\circ}$ journal and benefit from:}

- Convenient online submission

- Rigorous peer review

- Immediate publication on acceptance

- Open access: articles freely available online

- High visibility within the field

- Retaining the copyright to your article 